\title{
Discrete virus infection model of hepatitis B virus
}

\author{
Pengfei Zhang, Lequan Min* and Jianwei Pian \\ School of Mathematics and Physics, University of Science and Technology Beijing, \\ Beijing, 100083, PR China
}

\begin{abstract}
In 1996 Nowak and his colleagues proposed a differential equation virus infection model, which has been widely applied in the study for the dynamics of hepatitis B virus (HBV) infection. Biological dynamics may be described more practically by discrete events rather than continuous ones. Using discrete systems to describe biological dynamics should be reasonable. Based on one revised Nowak et al's virus infection model, this study introduces a discrete virus infection model (DVIM). Two equilibriums of this model, $E_{1}$ and $E_{2}$, represents infection free and infection persistent, respectively. Similar to the case of the basic virus infection model, this study deduces a basic virus reproductive number $R_{0}$ independing on the number of total cells of an infected target organ. A proposed theorem proves that if the basic virus reproductive number $R_{0}<1$ then the virus free equilibrium $E_{1}$ is locally stable. The DVIM is more reasonable than an abstract discrete susceptible-infected-recovered model (SIRS) whose basic virus reproductive number $R_{0}$ is relevant to the number of total cells of the infected target organ. As an application, this study models the clinic HBV DNA data of a patient who was accepted via anti-HBV infection therapy with drug lamivudine. The results show that the numerical simulation is good in agreement with the clinic data.
\end{abstract}

Keywords: Hepatitis B virus, modeling, discrete virus infection model, equilibrium stability, numerical simulation

\section{Introduction}

Hepatitis B is one of the serious infectious diseases which threaten to the global human health, and has become an important social and public health issue. Chronic hepatitis B virus (CHBV) infection can cause liver inflammation and fibrosis, and severe cases may develop into cirrhosis or liver cancer. HBV has infected nearly 2,000,000,000 people around the world, and no less than 240,000,000 persons become lifelong infection of HBV [1]. A chronic infection in liver, which probably develops into cirrhosis of the liver or liver cancer afterwards, can also be given rise to by HBV. Because of the acute or chronic effects of hepatitis B, approximately 780,000 people die every year [1].

In a series of previous researches on modeling virus infection dynamics, there exist two different kinds of mathematical models: the differential description of continuous time models [2-7] and the difference description of discrete time models [8-11].

Clinical-based mathematical dynamic modeling has an important significance for the study of the HBV infection. Reasonable HBV infection mathematical model can help to understand the virus infection,

\footnotetext{
${ }^{*}$ Address for correspondence: Lequan Min, School of Mathematics and Physics, University of Science and Technology Beijing, Beijing, 100083, P.R. China. Tel.: +8601062332803; Fax: +8601062332803; E-mail: minlequan@sina.com.
} 
clearance and evolution mechanism and laws, reveal some of the new features of HBV infection, evaluate new medical programs and make long-term efficacy prediction.

In previous researches [6,7,12-15], the mechanisms of virus infections have been described via differential equation models. Based on an HBV-infected patient's clinical data [2] with cell division mechanism, this study proposes a discrete HBV infection model, analyzes the stability of the disease free equilibrium, models and simulates the dynamics of the HBV-infected patient's anti-HBV infection treatment [2].

The structure of this paper is as follows: Section 2 presents the basic virus infection model and an amended model. Section 3 sets up the discrete virus infection model, and analyzes the first equilibrium of this model. Section 4 simulates the HBV-infected patient's dynamics of anti-HBV infection. Conclusions are made in Section 5.

\section{The basic and amended models}

Nowak and his colleagues' basic viral infection mathematical model (BVIM) has been widely used in learning the mechanisms of infection by some viruses [2], such as Human Immunodeficiency Virus (HIV) and hepatitis B, C virus:

$$
\left\{\begin{array}{l}
\frac{d x}{d t}=\lambda-d_{1} x-\beta v x \\
\frac{d y}{d t}=\beta v x-a y \\
\frac{d v}{d t}=k y-u v
\end{array}\right.
$$

here $x, y$ and $v$ represent the amount of uninfected target cells, infected target cells, and free virus, respectively. The constant producing rate of uninfected target cells is $\lambda$, death rate is $d_{1} x$, and the rate of uninfected target cells getting infected is $\beta v x$. The producing rate of infected target cells is $\beta v x$, and death rate is $a y$. The rate of free virus produced from infected target cells is $k y$, and death rate is $u v$. All parameters of Eq. (1) are all nonnegative numbers. Eq. (1) has an infection free equilibrium and an endemic persistent equilibrium. The basic virus reproduction number of Eq. (1) is $R_{0}=\lambda \beta k / a d_{1} u$.

Leenheer and Smith [14] have provided a global analysis approach and proved that if the basic virus reproduction number $R_{0}<1$, the infection free equilibrium is globally attractive and the virus will be cleared; if $R_{0}>1$, the endemic persistent equilibrium is globally attractive and the virus will be persisted in the target organs.

Observe that the terminology $\lambda / d_{1}$ is included in the basic virus reproduction number $R_{0}=\lambda \beta k / a d_{1} u$ of Eq. (1), which represents the sum of the cells of the organs. This can be understood that a person who has a larger target organ will be more likely to be infected by the virus. Therefore the basic virus infection Eq. (1) needs to be modified. In previous research [6,7,15], aiming at this irrationality, the authors use 
the standard incidence rate instead of the simple occurrence rate, and propose a modified model:

$$
\left\{\begin{array}{l}
\frac{d x}{d t}=\lambda-d_{1} x-\frac{\beta v x}{x+y} \\
\frac{d y}{d t}=\frac{\beta v x}{x+y}-a y \\
\frac{d x}{d t}=k y-u v
\end{array}\right.
$$

This model can deduce a reasonable basic virus reproduction number. It can be expressed as $R_{0}=$ $\beta k / a u$, which does not relevant to the total number of cells of a patient's infected organ. In our previous researches [5,6], we have already proved that if the basic virus reproductive number $R_{0}<1$, then the infection free equilibrium is globally attractive; if $R_{0}>1$, then the endemic persistent equilibrium is globally attractive.

\section{Discrete virus infection model}

\subsection{Model}

Biological evolution dynamic may be described more practically by discrete events rather than continuous ones. Based on the differential equation virus infection given in $[6,7]$, this study proposes the following assumptions.

(1) Liver cells live for $h$ days.

(2) The constant producing rate of uninfected liver cells is $\lambda$, the death rate is $d_{1} x$, and the rate of uninfected liver cells getting infected is $b v x /(x+y)$.

(3) The producing rate of infected liver cells is $b v x /(x+y)$, and death rate is ay.

(4) The rate of free virus produced from infected liver cells is $k y$, and death rate is $u v$.

Denote that the amount of uninfected liver cells on the $\mathrm{n}$-th day is $x(n)$, the amount of infected liver cells on the $\mathrm{n}$-th day is $y(n)$, and the amount of free virus on the $\mathrm{n}$-th day is $v(n)$, respectively. Then the amount of variable $x, y$ and $v$ on the $(n+1) t h$ day are described by the following discrete model.

$$
\left\{\begin{array}{l}
x(n+1)=x(n)+h\left[\lambda-d_{1} x(n)-\frac{b v(n) x(n)}{x(n)+y(n)}\right] \\
y(n+1)=y(n)+h\left[\frac{b v(n) x(n)}{x(n)+y(n)}-a y(n)\right] \\
v(n+1)=v(n)+h[k y(n)-u v(n)] .
\end{array}\right.
$$

The parameters in this Eq. (3) have the same meanings as those in Eq. (2). These seven parameters of Eq. (3) are all nonnegative numbers.

Model (3) possesses two equilibriums $E_{1}$ and $E_{2}$ :

$$
E_{1}=\left(\frac{\lambda}{d_{1}}, 0,0\right)
$$


represents the disease free steady state. $E_{1}$ is called virus free equilibrium.

$$
E_{2}=\left(\frac{\lambda}{d_{1}+a\left(R_{0}-1\right)}, \frac{\lambda\left(R_{0}-1\right)}{d_{1}+a\left(R_{0}-1\right)}, \frac{\lambda k\left(R_{0}-1\right)}{\mu\left[d_{1}+a\left(R_{0}-1\right)\right]}\right)
$$

represents the steady state of persistent infection. $E_{2}$ is called endemic equilibrium (persistent infection equilibrium). Here

$$
R_{0}=b k / a u .
$$

\subsection{Analysis the stability of equilibrium}

The local stability of the virus free equilibrium will be discussed in this section. Now we present some definitions and known results which will be useful in our investigation of Eq. (3).

\section{Preliminaries}

Let $F: \mathbb{R}^{n} \rightarrow \mathbb{R}^{n}$ be a continuous function, where $n$ is a non-negative integer. Consider the difference equation

$$
\begin{aligned}
& \boldsymbol{X}(k+1)= F(\boldsymbol{X}(k)), k=0,1, \ldots \\
& \boldsymbol{X}(k+1)=\left(\begin{array}{c}
x_{1}(1+1) \\
\cdot \\
\cdot \\
\cdot \\
x_{k}(k+1)
\end{array}\right)=\left(\begin{array}{c}
f_{1}(\boldsymbol{X}(k)) \\
\cdot \\
\cdot \\
\cdot \\
f_{k}(\boldsymbol{X}(k))
\end{array}\right) .
\end{aligned}
$$

with initial conditions $\boldsymbol{X}(0) \in \mathbb{R}^{n}$. Let $\overline{\boldsymbol{X}}$ be an equilibrium of Eq. (7), that is, $\overline{\boldsymbol{X}}=F(\overline{\boldsymbol{X}})$.

Denote

$$
J=\left(\begin{array}{cc}
\frac{\partial f_{1}(\boldsymbol{X})}{\partial x_{1}} & \ldots \frac{\partial f_{1}(\boldsymbol{X})}{\partial x_{n}} \\
\cdots & \cdots \\
\frac{\partial f_{n}(\boldsymbol{X})}{\partial x_{1}} & \cdots \frac{\partial f_{n}(\boldsymbol{X})}{\partial x_{n}}
\end{array}\right)_{\boldsymbol{X}=\overline{\boldsymbol{X}}} .
$$

the Jacobi matrix of Eq. (7) at the equilibrium $\bar{X}$.

The characteristic equation of Eq. (8) can be represented as

$$
\lambda^{n}-a_{n-1} \lambda^{n-1}-a_{n-2} \lambda^{n-2}-\cdots-a_{1} \lambda-a_{0}=0
$$


Theorem 3.1 (The Linearized Stability Theorem. For example see $[11,16])$.

(1) If all the absolute value of the eigenvalues of Eq. (9) are less than the unit 1, the equilibrium point $x$ of Eq. (7) is locally stable.

(2) If at least one eigenvalue's absolute value of Eq. (9) is not less than the unit 1, the equilibrium point $x$ of Eq. (7) is unstable.

Theorem 3.2 If $R_{0}<1$, then there exists a constant $h_{1}>0$ such that the virus free equilibrium point $Q_{1}$ of Eq. (3) is locally stable for all $h \in\left(0, h_{1}\right)$.

Proof of Theorem 3.2 By using the mathematical software (MATLAB), we get the Jacobian matrix of the Eq. (3).

$$
J=\left[\begin{array}{ccc}
-h d_{1}+1+h\left(a_{11}-a_{12}\right) & h\left(a_{11}\right) & -h\left(a_{13}\right) \\
-h\left(a_{11}+a_{12}\right) & -h a+1-\left(a_{11}\right) & h\left(a_{13}\right) \\
0 & h k & -h u+1
\end{array}\right]
$$

where

$$
a_{11}=\frac{b x v}{(x+y)^{2}}, a_{12}=\frac{b v}{x+y}, a_{13}=\frac{b x}{x+y}
$$

Hence

$$
J_{E_{1}}=\left[\begin{array}{ccc}
1-h d_{1} & 0 & -h b \\
0 & 1-h a & h b \\
0 & h k & 1-h u
\end{array}\right]
$$

So that

$$
\lambda E-J_{E_{1}}=\left[\begin{array}{ccc}
\lambda-1+h d_{1} & 0 & h b \\
0 & \lambda-1+h a & -h b \\
0 & -h k & \lambda-1+h u
\end{array}\right]
$$

Then the eigenvalues of Eq. (12) are

$$
\begin{aligned}
& \lambda_{1}=1-h d_{1}, \\
& \lambda_{2}=1-\frac{h}{2}\left((a+u)+\sqrt{(a-u)^{2}+4 b k}\right), \\
& \lambda_{3}=1-\frac{h}{2}\left((a+u)-\sqrt{(a-u)^{2}+4 b k}\right) .
\end{aligned}
$$


When $b k / a u<1$, we can prove $(a+u)-\sqrt{(a-u)^{2}+4 b k}>0$ holds.

Case 1.

If the following inequality holds, $0<h d_{1}<2$, that is, $h<2 / d_{1}$. Then we can obtain $\left|\lambda_{1}\right|<1$. Case 2.

If the following inequality holds,

$$
0<\frac{h}{2}\left((a+u)+\sqrt{\left.(a-u)^{2}+4 b k\right)}<2\right.
$$

that is,

$$
h<\frac{4}{(a+u)+\sqrt{(a-u)^{2}+4 b k}} .
$$

Then $\left|\lambda_{2}\right|<1$.

Case 3.

If the following inequality holds,

$$
0<\frac{h}{2}\left((a+u)-\sqrt{\left.(a-u)^{2}+4 b k\right)}<2\right.
$$

that is,

$$
h<\frac{4}{(a+u)-\sqrt{(a-u)^{2}+4 b k}} .
$$

Then $\left|\lambda_{3}\right|<1$.

Consequently, when $R_{0}=b k / a u<1$, then we can obtain $-1<\lambda_{1,2,3}<1$, if

$$
0<h<h_{1}=\min \left(\frac{2}{d_{1}}, \frac{4}{(a+u) \pm \sqrt{(a-u)^{2}+4 b k}}\right) .
$$

Therefore, $E_{1}$ is locally stable and this completes the proof.

In the process of actual calculation, since $a=d_{1}=3.79 \times 10^{-3}, u=0.67, b k=a u R_{0}<R_{0}<$ $200, h \approx 1.6 \times 10^{-4}$ [17], we conclude (see the next section) that the following inequality holds:

$$
h<\min \left(\frac{2}{d_{1}}, \frac{4}{(a+u) \pm \sqrt{(a-u)^{2}+4 b k}}\right) .
$$


Table 1

Evolution of the selected patient's plasma HBV DNA loads (log copies/ml). Here VL represent the practical virus levels, and SM represent the numerical simulations.

\begin{tabular}{llllllllllllllll}
\hline Week & 0 & 2 & 4 & 6 & 8 & 12 & 16 & 20 & 24 & 28 & 32 & 36 & 40 & 44 & 48 \\
\hline VL & 7.90 & 6.08 & 6.02 & 6.03 & 6.02 & 6.03 & 6.02 & 6.03 & 6.02 & 7.90 & 7.90 & 7.90 & 7.90 & 7.90 & 7.85 \\
SM & 7.90 & 6.14 & 6.11 & 6.10 & 6.07 & 6.03 & 5.99 & 5.94 & 5.90 & 7.76 & 7.83 & 7.87 & 7.89 & 7.90 & 7.90 \\
\hline
\end{tabular}

\section{Dynamics simulation of one patient's anti-HBV infection treatment}

This section will simulates one HBV infected patient's dynamics for anti-HBV infection therapy with drug lamivadine [2]. The experiment consists of a 24-week treatment and a 24-week follow-up without treatment.

The selected patient's (numbered by 2) plasma HBV DNA data are presented from Figure 1 (B) in [2]. Observe that when withdrawing the drug, the patient's plasma HBV DNA loads reduce quickly (also see Table 1 and Figure 1 in this paper). Since the patient's ALT levels are not abnormal in the treatment [2], it suggests that his immune system is not fully activated.

Therefore one can assume, from Eq. (3), that the discrete anti-HBV infection dynamic model of the patient treated with drugs can be described by

$$
\left\{\begin{array}{l}
x(n+1)=x(n)+h\left[\lambda-d_{1} x(n)-(1-m) \frac{b v(n) x(n)}{x(n)+y(n)}\right] \\
y(n+1)=y(n)+h\left[(1-m) \frac{b v(n) x(n)}{x(n)+y(n)}-a y(n)\right] \\
v(n+1)=v(n)+h[(1-n) k y(n)-u v(n)]
\end{array}\right.
$$

here $m, n$ are the effectiveness of the treatment. Before the patient's anti-HBV infection treatment, one assumes that $m=n=0$, and his HBV DAN level is in the virus persistent state $E_{2}$. Consequently

$$
k=u v(0)\left[d_{1}+a\left(R_{0}-1\right)\right] /\left[\lambda\left(R_{0}-1\right)\right], b=a u R_{0} / k .
$$

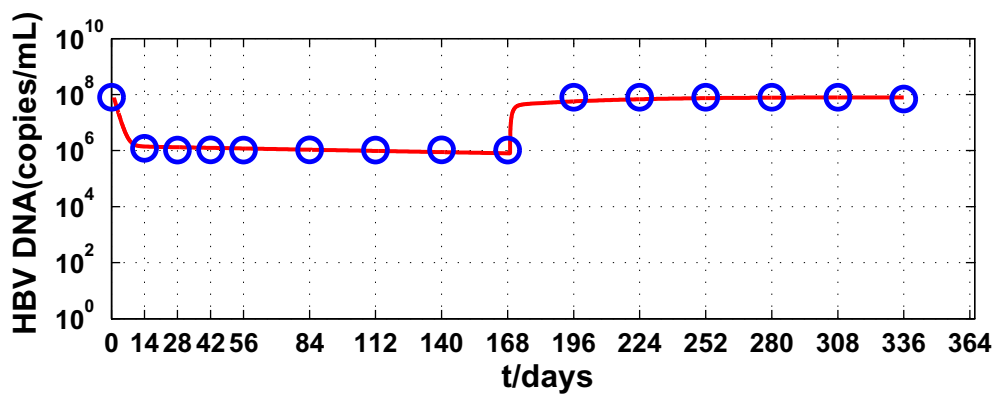

Fig. 1. Blue circle dots represent the clinical data points. And the red solid line means the dynamic numerical simulation of the treatment model. 
The detail steps in relation to the calculation of model's coefficients are as follows.

1) A human liver has about $2 \times 10^{11}$ liver cells [17], and contains about $3000 \mathrm{ml}$ plasma in total. In this paper, the qualities of tested virus per milliliter are in copies. This suggests

$$
\lambda / d_{1} \approx 2 \times 10^{11} / 3000 .
$$

2) Because the half-life of a liver cell is around 6 months, one may hypothesize

$$
d_{1}=-\ln (0.5) / 183 \approx 0.00379 .
$$

3) Choose $\mu=0.67$ [17], which equals to hypothesis that around one day is the half-life of a virus.

4) We select stepsize

$$
h=\frac{1}{\frac{1 \times 10^{11}}{183 \times 24 \times 60 \times 60}} \approx 1.6 \times 10^{-4},
$$

which is equivalent to assume that the half-life of a hepatocyte is around 183 day.

5) Hypothesize that prior to the lamivudine therapy, the patient is in the state of stable virus persist infection, which is,

$$
(\bar{x}, \bar{y}, \bar{v})=E_{2}=\left(\frac{\lambda}{d_{1}+a\left(R_{0}-1\right)}, \frac{\lambda\left(R_{0}-1\right)}{d_{1}+a\left(R_{0}-1\right)}, \frac{\lambda k\left(R_{0}-1\right)}{u\left[d_{1}+a\left(R_{0}-1\right)\right]}\right) .
$$

6) With the clinical data and numerical simulation, one is able to determine the parameters below.

$$
\left\{d_{1}, a, u, R_{0}, m, n\right\}=\{0.00379,0.003 .79,0.67,9,0,0.982\} .
$$

Substituting the above parameters into Eq. (19), the numerical simulating of Eq. (19) is presented in Figure 1. The simulated data are provided in Table 1. Observe that the simulated results agreed well to the patient's clinical data (see the circles shown in Figure 1). Before the lamivudine treatment, the baseline basic virus reproduce number is $R_{0}=9$ (when $m=0$, and $n=0$ ). $R_{0}$ will drop to 0.162 during the therapy. After stopping the therapy, the patient's plasma HBV DNA load returned quickly to the state prior to the treatment. 


\section{Conclusions}

Virus infection is a primary global health issue. Building mathematical virus infection models is important for the understanding of complicated phenomena in scientific terms. The cell fissions and virus replications should be discrete events rather than continuous ones. The evolution dynamics of virus infection may be described more reasonablely by discrete models rather than continuous models.

Based on previous researches [6,7], this study proposes a discrete difference equation model to describe virus infection dynamics. Theorem 3.2 in this paper gives the condition that the virus free equilibrium point of our model is locally stable. The basic virus reproductive number is independent on the number of total cells of the infection target organ. Therefore, it is more reasonable than the model proposed by Nowak et al.[2].

An instance shows that the theoretical analysis and numerical results are capable to facilitate comprehending HBV infection and anti-HBV infection treatment. The research on global attractivity of equilibrium point is the further issue on the study of virus infection dynamics.

\section{Acknowledgement}

The authors gratefully acknowledge anonymous reviewers for very valuable comments. This project is supported by the National Natural Science Foundation of China (Grant Nos. 61074192).

\section{References}

[1] World Health Organization. Hepatitis B[OL], March 2015, http://www.who.int/mediacentre/factsheets/fs204/en/.

[2] M.A. Nowak, S. Bonhoeffer, A.M.Hill, et al., Viral dynamics in hepatitis B virus infection, Proceedings of the National Academy of Sciences 93 (1996), 4398-4402.

[3] L. G. Guidotti, R. Rochford, J. Chung, et al., Viral clearance without destruction of infected cells during acute HBV infection, Science 284 (1999), 825-829.

[4] J. M. Murray, R. H. Purcell and S. F. Wieland, The half-life of hepatitis B virions, Hepatology 44 (2006), 1117-1121.

[5] S.A. Gourley, Y. Kuang and J.D. Nagy, Dynamics of a delay differential model of hepatitis B virus, Journal of Biological Dynamics 2 (2008), 140-153.

[6] L. Min, Y. Su and Y. Kuang, Mathematical analysis of a basic model of virus infection with application to HBV infection, Rocky Mountain Journal of Mathematical 38 (2008), 1573-1584.

[7] Y. Zheng, L. Min, Y. Ji, et al., Global Stability of Endemic Equilibrium Point of Basic Virus Infection Model with Application to HBV Infection, Journal of Systems Science and Complexity, 23 (2010), 1221-1230.

[8] L. J. S. Allen, Some discrete-time SI,SIR, and SIS epidemic models, Journal of Mathematical Biology 124 (1994), 83-105.

[9] D. Zhang and B. Shi, Oscillation and global asymptotic stability in a discrete epidemic model, Journal of Mathematical Analysis and Applications 278 (2003), 194-202.

[10] Y. Muroya, Y. Nakata, G. Izzo, et al., Permanence and global stability of a class of discrete epidemic models, Nonlinear Analysis: Real World Applications 12 (2011), 2015-2117.

[11] Zengyun Hu, Zhidong Teng and Haijun Jiang, Stability analysis in a class of discrete SIRS epidemic models, J. Nonlinear Analysis: Real World Applications 13 (2012), 2017-2033.

[12] A Neumann, N. Lam, H. Dahari, et al., Hepatitis C viral dynamics in vivo and antiviral efficacy of the interferon- therapy, Science 282 (1998), 103-107.

[13] S. Zeuzem, J.M. Schmidt, J.H. Lee, et al., Effect of inferferomalt on the dynamics of Hepatitis C virus turnover in vivo, Hepatology 23 (1996), 366-371.

[14] P. D. Leenheer and H. L. Smith, Virus dynamics: A global analysis, SIAM Journal on Applied Mathematics 63 (2003), 1313-1327.

[15] Y. Zheng, L. Min, X. Chen, et al., Dynamics Analysis of HBV Infection Model with Prediction of Long-terms Therapy Results, Hepatology International, 5 (2011), 523.

[16] Q. wang, F. Zeng, X. Liu, et al., Stability of a rational difference equation, Applied Mathematics Letters 25 (2012), 2232-2239.

[17] M.A. Nowak and R.M. May, Viral Dynamics, Oxford University Press, Oxford, 2000, pp. 53-58. 